\title{
Heterotopías urbanas. Modalidades e innovaciones en la producción del espacio estatal porteńo
}

Pablo Elinbaum. Consejo Nacional de Investigaciones Científicas y Técnicas (CONICET), Buenos Aires, Argentina.

RESUMEN | Las heterotopías urbanas son lugares que interrumpen la aparente continuidad y normalidad de la ciudad. Por ejemplo, son las villas miseria y favelas sudamericanas, pero también los glamorosos distritos de elite, como el emblemático Canary Wharf en Londres. ¿Cómo se incorporan estos espacios singulares a la espacialidad supuestamente unitaria y equilibrada del Estado? A partir de la comparación de los planes parciales implementados para desarrollar simultáneamente dos "villas" de Buenos Aires en un mismo territorio -la Comuna 8-, en este artículo se indaga en los entramados de proyectos y estrategias para mostrar las modalidades e innovaciones en la producción del espacio estatal porteño. A partir de un enfoque cualitativo, apoyado en dos dimensiones básicas del planeamiento urbano, se evidencian algunas relaciones dialécticas entre diferentes escalas, ciclos de inversión y regulación que suelen ser soslayadas no solo por las investigaciones acotadas a los "enclaves", sino también por los efectos discursivos de las políticas urbanas pretendidamente "integrales".

PALABRAS CLAVE | renovación urbana, proyecto urbano, campamentos.

ABSTRACT | Urban heterotopies are places that interrupt the apparent continuity and normality of the city. For example, they are the shanty towns, but also the glamorous elite districts such as Canary Wharf in London, to cite a well-known case. How are these spaces embedded into the supposedly unitary and balanced state spatiality? Based on the comparative case study of the sector plans for two "villas" in Buenos Aires, implemented simultaneously in the same territory-and during the same urbanization process-this article investigates the networks of projects and strategies to show the modalities and innovations in the production of the state space of Buenos Aires. Based on a qualitative approach for two basic planning dimensions, the paper shows dialectical relationships, between different scales, investment and regulation cycles that are usually overlooked not only by research, delimited to the "enclaves", but also due to the discursive effects of the supposedly "integral" urban policies.

KEYWORDs | urban renewal, urban project, slums. 


\section{Introducción}

Los Grandes Proyectos Urbanos (GPU) (Urban Megaprojects, en su acepción anglosajona) son hoy, sin lugar a dudas, el tipo de intervención que mejor representa el urbanismo neoliberal de los últimos treinta años. Según una definición más o menos consensuada, los GPU son operaciones de renovación de gran escala, generalmente impulsadas a través de acuerdos mixtos entre el sector público y el privado, y modelos de gestión empresariales basados en el objetivo de revalorizar el suelo urbano (Jajamovich \& Kozak, 2019). Estos emprendimientos han sido profusamente abordados desde diversas perspectivas teóricas e ideológicas, incluidas aquellas que los proponen como modelos de gestión ideal, hasta otras que señalan todas sus desventajas y se pronuncian en su contra (Jajamovich, 2019). Desde estos enfoques, los GPU han sido estudiados en tanto herramientas de captación de plusvalías inmobiliarias (Smolka \& Furtado, 2001), como estrategias de renovación urbana (Lungo, 2004), como motores de procesos de gentrificación (Janoschka, 2016), como estrategias de marketing de ciudades (Silvestre, 2017) y como modelos de gestión del suelo (Cuenya, 2012).

Pese a la marcada diversidad de enfoques, en la gran mayoría de las investigaciones se suele conceptualizar los GPU, a nivel micro, como enclaves y, a nivel macro, como metáforas de la globalización. La opción teórica de concebirlos como fragmentos urbanos evidencia un sustrato cognitivo que no es neutral, ya que tiene consecuencias no menores en la epistemología sobre este tipo de política urbana. Este modo particular de enfocar el problema, sin alzar la mirada más allá de la parcela intervenida o analizada, es también frecuente en las intervenciones y estudios sobre los espacios informales. Denominados indistintamente como "precarios", "marginales" o "periféricos", al diferenciarse de la trama urbana regular de la ciudad, estos territorios son percibidos generalmente como heterotopías: lugares que interrumpen la aparente continuidad de los espacios ordinarios de la vida cotidiana, dando por sentado, en esa apreciación, una "normalidad" y un "orden previo", como señalaba Foucault en su célebre ensayo "De los espacios otros" (Foucault, 1967).

La noción de heterotopía es relativamente nueva en los estudios urbanos. Entre la incipiente bibliografía cabe mencionar la compilación de Michiel Dehaene y Lieven De Cauter, Heterotopia and the City (2008) como una primera exploración sistemática del alcance de este concepto para describir las ciudades europeas contemporáneas. En el contexto latinoamericano también se ha utilizado esta noción, sobre todo en el campo de la historia, como un instrumento epistemológico para revisar algunos fenómenos urbanos y arquitectónicos excepcionales -o desaparecidos-, enfatizando las relaciones entre cultura y espacio (Caride \& Dal Castello, 2015). Algunos geógrafos brasileńos, por su parte, han utilizado el concepto de heterotopía para estudiar los lugares que escapan del alcance de la dinámica capitalista (Santos, 2014; Tramontani, 2010), los modos de resignificar el espacio público (Valverde, 2009), los ámbitos del poder y del contrapoder, la normatividad y la resistencia (Fernández, 2017). Retomando estas últimas indagaciones, el punto de partida de este artículo radica en problematizar el abordaje parcial y selectivo que se les da a los grandes proyectos urbanos y a las villas miseria desde la lógica del planeamiento. 
Se trata de poner en evidencia el entendimiento limitado sobre la producción del espacio estatal y sobre los alcances e implicancias, proyectuales y estratégicas, de la articulación de las políticas públicas aisladas en el marco de un mismo proceso de renovación urbana.

El análisis de la convergencia de diferentes planes y proyectos es una oportunidad para clarificar no solo las modalidades e innovaciones en la producción del espacio estatal, sino, también, para revisar la propia concepción de los GPU y de los espacios informales. De este modo, se pretende enfatizar algunas relaciones no tan evidentes, dinámicas y complementarias, entre diferentes espacios y escalas territoriales, que suelen omitirse tanto en las investigaciones enfocadas en unidades de estudio estancas, como también en las políticas urbanas pretendidamente "integrales". ¿Qué papel desempeńa el planeamiento urbano en la producción del espacio estatal? ¿Cómo se relacionan los grandes desarrollos inmobiliarios y la reurbanización de las villas miseria concurrentes en un mismo territorio? ¿Cómo pueden concebirse estos episodios urbanos singulares como campos de la acción estatal en el espacio? ¿Por qué se suele hablar de gestión integral del territorio cuando, al mismo tiempo, se crean instrumentos y espacios cada vez más fragmentados y superpuestos?

Responder a estos interrogantes es importante para tomar distancia del punto de vista que da por sentado que el planeamiento urbano es una disciplina exclusivamente técnica, relativamente autónoma y ajena a la reproducción de los regímenes políticos y económicos. Se trata de ofrecer una lectura posible acerca del lugar específico que ocupa la práctica del urbanismo en los Estados capitalistas contemporáneos, evidenciando su papel clave en la producción de la forma espacial del territorio estatal. El objetivo de este artículo apunta a explicitar esas relaciones, ofreciendo una interpretación que evidencie los entramados de proyectos y estrategias espaciales a través de los cuales se producen nuevas territorialidades. De forma particular, este objetivo pretende contribuir a la epistemología de la producción del espacio, considerando diferentes enfoques combinados, como la teoría del Estado, la aproximación estratégica relacional y la teoría crítica del planeamiento urbano. Asimismo, se pretende indagar en el análisis empírico del espacio estatal, a través del estudio de los procesos y las formas que lo constituyen.

Los objetivos planteados se apoyan en un abordaje metodológico cualitativo, a través del estudio de caso de dos proyectos concurrentes en la Comuna 8 de la Ciudad Autónoma de Buenos Aires (CABA), uno implementado para desarrollar el conjunto residencial de la Villa Olímpica y el otro para reurbanizar el asentamiento informal denominado Villa 20. El análisis se centrará en la interrelación de dos dimensiones clave del planeamiento urbano: la instrumental y la socioespacial. Asimismo, se considerarán dos unidades de análisis: la comunal y la de los ámbitos de planeamiento parcial o por sector. La opción por este doble acercamiento pretende evidenciar no solo las causalidades entre las dimensiones y las escalas de análisis, sino, sobre todo, mostrar los sutiles procesos de reescalamiento de las políticas públicas y el carácter estratégico de los proyectos estatales. En cuanto a las fuentes, el artículo se nutre del análisis de diversos documentos oficiales (planes, proyectos, normas, convenios, reglamentos, etcétera), de investigaciones antecedentes sobre los casos de estudio (en particular, Gil et al., 2011 y Roitman, 2016), la realización 
de entrevistas a informantes clave, y la creación de planos y mapas ad hoc, contribuyendo con nuevas fuentes primarias a la explicación de las heterotopías y de los espacios estatales.

El artículo se estructura en cuatro partes. Después de la introducción, en el segundo apartado, se describe el marco conceptual y el metodológico. En la tercera parte, se desarrolla el análisis empírico de las dos "villas". Y, en las dos últimas, se cruzan y discuten los resultados, para concluir con una serie de reflexiones convergentes en los casos, sugiriendo posibles caminos para las futuras investigaciones.

\section{Planeamiento urbano y producción de espacio estatal}

Durante los últimos treinta años se consolidó un nuevo paradigma en los estudios urbanos, a partir de los diversos giros espaciales que cruzaron transversalmente varias disciplinas al punto de desmembrarlas. Cada uno de ellos reveló asunciones tácitas y a menudo problemáticas en la base de las ciencias sociales que permitieron elaborar un mejor entendimiento del espacio a partir de la reflexión crítica sobre cuatro nociones clave: lugar, territorio, escala y red (Dicken et al., 2001; Paasi, 2004; Sheppard, 2002). La relevancia de estos debates se refiere a los esfuerzos por descifrar los cambios en los procesos de urbanización a escala planetaria, particularmente aquellos vinculados a la crisis del fordismo, la intensificación de "lo global", la reestructuración de las geografías heredadas de la acumulación capitalista, la regulación estatal y las luchas sociopolíticas (Jessop et al., 2008). Si bien estos giros y conceptos espaciales problematizan cuestiones específicas, como se explica a continuación, están plenamente imbricados en términos teóricos y empíricos.

A principios de los ochenta, el primero de estos giros espaciales se enfocó en problematizar el concepto de "lugar". Estudiados tradicionalmente como unidades fijas que ocupan un área acotada, los lugares comenzaron a pensarse cada vez más como espacios constituidos de forma relacional, solapados en procesos polivalentes y en tramas sociales más amplias (Cresswell, 2004; Hudson, 2002; Massey, 1984, 1994). A finales de la década del ochenta, la noción de "territorio" pasó a ser el centro de la segunda instancia de discusión teórica, movilizada por la exploración de la geometría variable de las regiones y los vectores de desarrollo tensionados por las dinámicas metropolitanas y globales. Entre otras cuestiones, se criticó el entendimiento de la territorialidad del poder político del Estado concebido como un espacio acotado a las jurisdicciones nacionales (Brenner et al., 2003). A partir de los noventa se vivió un controvertido giro en torno a la noción de "escala" que se enfocó en descifrar cómo las relaciones globales, nacionales y locales heredadas del periodo fordista estaban siendo calibradas a través de la reestructuración posindustrial del capitalismo y la racionalización del Estado. Recuperando varios de los postulados de Lefebvre, a través de esta nueva aproximación teórica se pretendía comprender no solo los procesos de producción de las escalas, sino también su estabilización (scalar $f(x)$ y los modos en que las políticas nacionales saltean ciertos niveles de gobierno tergiversando la subsidiariedad administrativa (scale jump). La revisión epistemológica de las escalas fue fundamental para explicar sus impactos en el solapamiento y rejerarquización de las estructuras socioambientales, entre la lógica 
de las instituciones estatales, las economías capitalistas, los regímenes de la ciudadanía y los sistemas urbanos (Brenner, 1998, 2000; Collinge, 1999; Smith, 1995; Swyngedouw, 1997). Más recientemente, algunos académicos se han enfocado en discutir la noción de "red", estudiando las formas transversales y rizomáticas de la conectividad interespacial (Amin, 2002; Castells, 1996; Graham \& Marvin, 2001; Taylor, 2004). A partir de este cuarto giro en la teoría del espacio, las investigaciones sobre las geografías de las redes se han robustecido en varios campos de las ciencias sociales, tales como los estudios de las cadenas de mercancías, las dependencias intraempresariales, los sistemas de gobernanza, las relaciones interurbanas y los vínculos entre el Estado y los movimientos sociales (Grabher, 2006).

Las reflexiones teóricas resultantes de los diferentes giros espaciales y de los debates acerca de las nociones de lugar, territorio, escala y red constituyen un marco de conocimiento innovador para explorar los modos en que el planeamiento urbano produce el espacio estatal. Pero antes de pasar a las evidencias empíricas, parece oportuno revisar algunas definiciones básicas sobre este concepto. La idea de "espacio estatal" fue desarrollada inicialmente en el libro New State Spaces (2004) en el que Neil Brenner reformula en términos espaciales tres nociones clave de la teoría del Estado del politólogo inglés Robert Jessop (2001). La "forma espacial del estado", señala Brenner en el texto citado, se refiere al principio de la territorialidad concebida sobre la base de unidades políticas formalmente equivalentes, moduladas y autónomas, tales como los países, las provincias y los municipios. Esta mirada formalista es la piedra angular del segundo concepto, "proyecto espacial", que se refiere a aquellas iniciativas que tienen como fin diferenciar y, al mismo tiempo, integrar la territorialidad del Estado en jurisdicciones y niveles administrativos institucionalizados. Su objetivo apunta a coordinar las regulaciones urbanísticas, los vínculos fiscales, la provisión de servicios, la inversión en infraestructuras, etcétera. En contraposición, Brenner destaca la noción de "estrategia espacial" que se refiere a aquellas iniciativas del Estado que tienen como fin privilegiar el desarrollo económico y social de algunos territorios por sobre otros, haciendo de la selectividad una de las características inherentes de la acción estatal.

Tanto los proyectos como las estrategias se apoyan en discursos que tienden a retratar a las instituciones estatales como si fuesen entidades coherentes y estables. En palabras de Brenner (2004), estos discursos producen "efectos de estado" que transmiten la apariencia tranquilizadora con la cual solemos percibir a las instituciones estatales. Apariencia que está lo suficientemente arraigada y naturalizada como para soslayar las desigualdades históricas y territoriales producidas tanto por el desarrollo urbano en los Estados capitalistas, como por las luchas sociopolíticas que tensionan la supuesta estabilidad de la forma y de las instituciones estatales.

\section{Dos dimensiones clave del planeamiento urbano}

A partir de los conceptos comentados en el apartado anterior y retomando los objetivos formulados en la introducción, en este punto se describen las categorías y dimensiones analíticas con las cuales se intentará describir cómo se produce el espacio estatal a través del planeamiento urbano. Con este fin, el análisis se enfocará 
en la combinación de dos dimensiones básicas del planeamiento: la socioespacial y la instrumental.

Comenzando por el enfoque instrumental, más allá de los diferentes contextos de diseño e implementación, los planes urbanos pueden clasificarse a partir de dos tipos: los generales y los parciales (Esteban, 1998). Los primeros combinan una amplia gama de temas y consideran como ámbito de intervención las jurisdicciones completas, ya sea un municipio, una provincia o un país. Por su parte, los planes parciales tienen un contenido acotado, más bien sectorial, y afectan solo a una porción del territorio, que suele delimitarse de forma ad hoc. Los planes urbanos también se caracterizan por su alcance, que puede ser vinculante o indicativo cuando sus prescripciones no son obligatorias (Faludi \& van der Valk, 1994). Además del efecto que producen, su operatividad instrumental también depende de las representaciones espaciales y de los discursos (Elinbaum \& Massidda, 2020; Van Duinen, 2004). Por su carácter estratégico -entre la coordinación y la cooperación-, las representaciones son fundamentales no solo para explicar los proyectos durante la redacción de los planes urbanos (Faludi, 1996; Neuman, 1998), sino también para argumentar la territorialidad y las múltiples escalas de los espacios estatales, tal como se da, por ejemplo, en el fraccionamiento o en la fusión de las jurisdicciones históricas, en la creación -o anulación- de niveles administrativos, en la expropiación de parcelas privadas, en la reclasificación del suelo rural en urbano, en la catalogación patrimonial, etcétera. De este modo, los espacios estatales presentan huellas superpuestas de los varios proyectos e instrumentos que los produjeron y, al mismo tiempo, evidencian las estrategias de intervención política y económica que motivaron su diseño e implementación.

La segunda dimensión que se utilizará para estudiar el planeamiento es la socioespacial. A través de este enfoque se analiza cómo los planes urbanos producen la territorialidad estatal, combinando diferentes principios de organización y estructuración espacial, integrando los cuatro conceptos ya mencionados más arriba (territorio, escala, lugar y red). Tal como los define Jessop (2001), estos principios de interacción pueden entenderse como expresiones de diversos intentos de coordinación estratégica y articulación estructural, dentro de contextos espaciales y temporales específicos y según una aproximación estratégica-relacional (strategic-relational approach). A través de este encuadre teórico y metodológico se intenta mostrar, por un lado, las contradicciones, dilemas y conflictos que caracterizan a las formaciones sociales capitalistas en etapas y coyunturas específicas. Por otro lado, se pretende evidenciar cómo estas contradicciones se resuelven o, al menos, se desplazan con el fin de regular y gobernar la acumulación de capital y la dominación política (Jessop \& Sum, 2006). De este modo, la aproximación estratégica-relacional permite indagar en las varias formas de reestructuración socioespacial a través de algunas de sus categorías fundamentales ya mencionadas, como la selectividad estatal, las estrategias de acumulación y los proyectos hegemónicos.

Desde este punto de vista, las intervenciones del planeamiento urbano ya no pueden concebirse como hechos aislados o emprendimientos que se inician desde cero, sino como intervenciones atadas a la inercia de las decisiones tomadas en el pasado (path dependence) y a la permanencia de los desequilibrios socioespaciales, 
históricos y estructurales. En este sentido, cabe retomar la noción de rounds de inversión y de regulación, acuñada por Doreen Massey (1985), como una metáfora oportuna para descifrar cómo se materializa la geografía económica del capitalismo, cómo se produce la espacialidad resultante de la división del trabajo y, en particular, cómo los proyectos y los planes urbanos actúan como catalizadores de los sucesivos modelos económicos y ciclos de inversión en las infraestructuras que, de forma gradual e impredecible, se superponen sobre las geografías históricas y los arreglos espaciales heredados.

\section{Dos villas, un territorio: la producción del espacio estatal en la Comuna 8 de la Ciudad de Buenos Aires}

Sobre la base de las orientaciones teóricas y metodológicas desarrolladas más arriba, en este apartado se intenta explicar y discutir las aproximaciones proyectuales y estratégicas del planeamiento en su misión de incorporar las heterotopías urbanas en el espacio estatal. Para eso, el análisis se apoya en el estudio de dos planes parciales implementados para desarrollar dos "villas", la Olímpica y la Veinte, ubicadas en la Comuna 8 de la Ciudad Autónoma de Buenos Aires (Figura 1). En este caso, el juego de palabras "dos villas" se refiere al uso que en Argentina se le da al término "villa" como asentamiento informal (cf. Ziccardi, 1977) y al uso más universal de "villa” -sin esa asociación- aplicado a los predios donde se desarrollan las olimpiadas.

FIgURA I | Mapa de situación de los dos planes parciales a escala de la CABA y de la Comuna 8
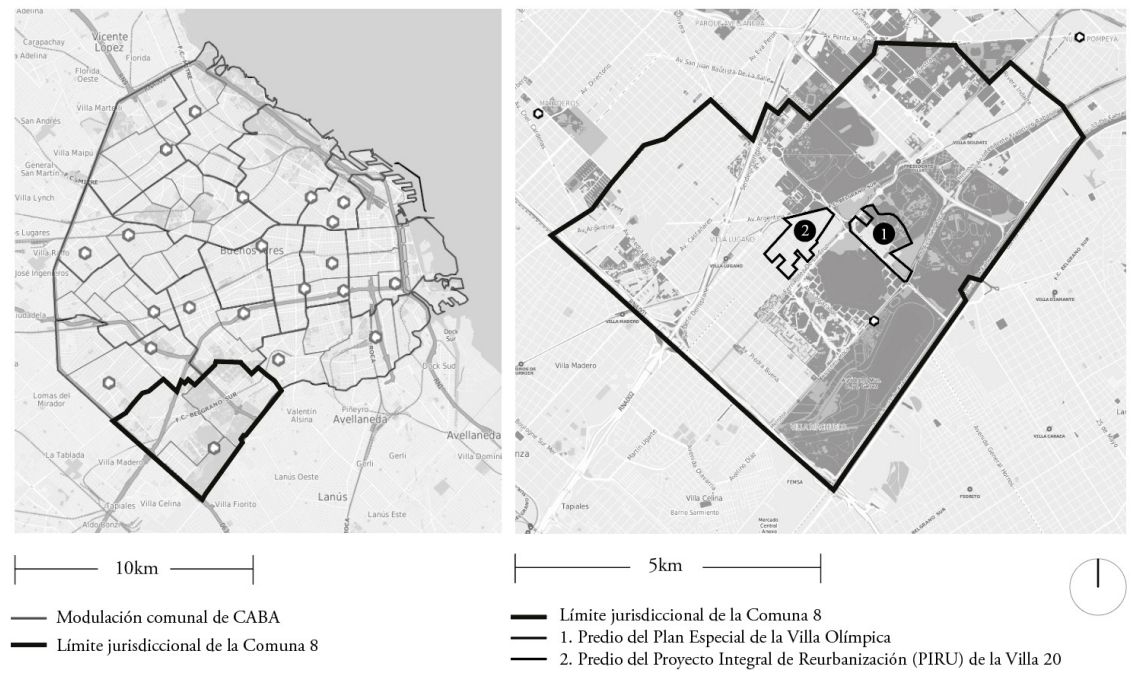

FUENTE: ELABORACIÓN PROPIA A PARTIR DE LA CARTOGRAFÍA OFICIAL DISPONIBLE ON-LINE EN EL SITIO WEB DEL GOBIERNO DE LA CIUDAD 


\section{Un nuevo estilo de planeamiento}

En términos de desarrollo urbano, el territorio que hoy ocupa la Comuna 8 ha sido históricamente el más rezagado de la Ciudad de Buenos Aires. Sin embargo, el estancamiento en los índices de calidad de su espacio público, sus servicios y equipamientos contrasta con su dinamismo territorial evidente en los continuos cambios de la normativa urbanística. En el Código de Edificación de 1959, los terrenos donde hoy se ubican la Villa Olímpica y la Villa 20 fueron concebidos con el uso de "urbanización parque"; pocos años después, en el marco del Plan Especial Parque Almirante Brown de 1962, fueron reclasificados como "áreas de promoción" y "espacios deportivos"; más tarde, en el Código Urbano de 1977, los dos predios quedaron incluidos en unidades de gestión más pequeñas, los denominados "equipamientos especiales" que hasta hoy son una categoría tan ambigua que admite prácticamente cualquier uso.

A partir de ese momento, se aplicó sobre el código urbano un sinnúmero de "interpretaciones" y "excepciones" naturalizadas como el proceder rutinario que caracteriza al urbanismo porteño desde hace más de cuarenta ańos. Con esta lógica, tras la aprobación de la Ley 5704 de 2016, se modificó la afectación de varios polígonos delimitados dentro del predio que, hasta ese momento, había sido el Parque de la Ciudad (Figura 2). Estas modificaciones se realizaron con el fin de promover el desarrollo inmobiliario en la Comuna 8, fijando indicadores suficientemente lucrativos como para atraer al sector inmobiliario e impulsar el proceso de renovación urbana. En este sentido, una de las primeras iniciativas del Estado fue la de construir la Villa Olímpica concibiéndola a partir de algunos elementos propios de los grandes proyectos urbanos. El primero, la modulación del predio en unidades de gestión, habilita la participación de diferentes empresas promotoras y, al mismo tiempo, permite que el barrio se construya por etapas. Se reduce, de este modo, el riesgo de inversión, en la medida en que el Estado garantiza previamente la ejecución de las infraestructuras, los equipamientos, los servicios, la seguridad, etcétera. El segundo elemento que se rescata de los grandes proyectos urbanos se refiere a la voluntad de aislar el barrio del entorno precario poco favorable para la promoción inmobiliaria. Si bien las viviendas de la Villa Olímpica no fueron concebidas para un target de elite, la tipología definida para los departamentos de clase media responde a un claro objetivo rupturista con el entorno socioeconómico dominado por el hábitat popular.

De forma complementaria, el marco de los Juegos Olímpicos de la Juventud 2018 le permitió al Gobierno de la Ciudad justificar la iniciativa para privatizar 40 hectáreas de suelo público, argumentando la necesidad de construir las 1.400 viviendas necesarias para alojar a los deportistas durante un mes. Lo más novedoso, sin embargo, es que el gobierno se encargó también de comercializar las viviendas, asumiendo el doble papel de promotor y agente inmobiliario. O, dicho de otro modo, se abstuvo de crear plusvalías, excluyendo del mercado la primera camada de viviendas. En cambio, las otras unidades de gestión se concibieron, de forma más convencional, como grandes parcelas con servicios e indicadores precisos para ser desarrolladas por los promotores inmobiliarios (Figura 2). 
Figura 2 | Predio de la Villa Olímpica presentado en el concurso que organizó la Sociedad Central de Arquitectos (sCA)

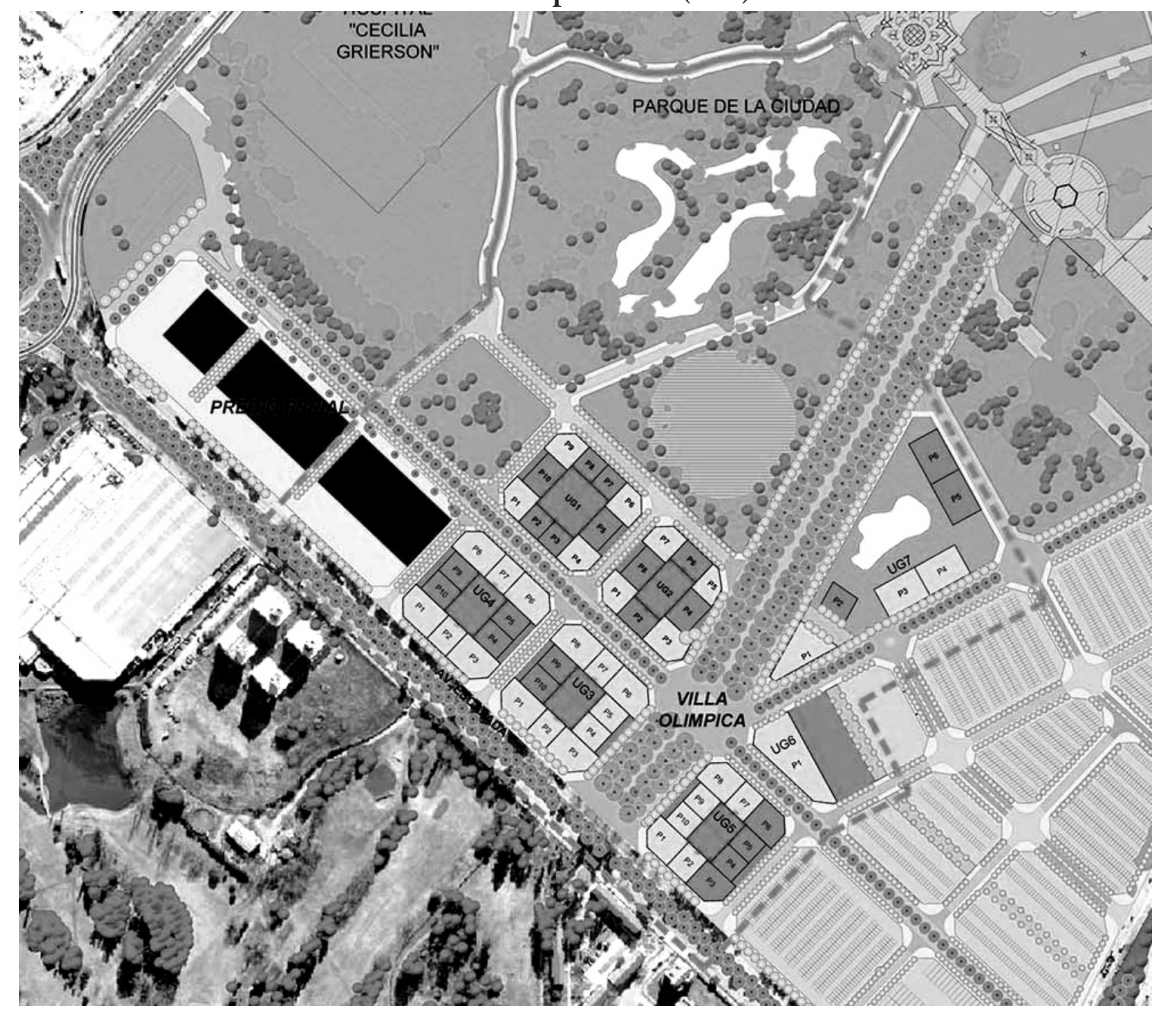

NOTA: EN GRIS CLARO, SE INDICAN LAS UNIDADES DE GESTIÓN COMERCIALIZADAS POR EL GOBIERNO DE LA CIUDAD

FUENTE: SCA.HTTP://SOCEARQ.ORG/2.0/20I4/IO/O7/CONCURSO-NACIONAL-DE-ANTEPROYECTOS-VIVIENDAS -PARA-LA-VILLA-OLIMPICA-QUINTO-CONCURSO/

A diferencia del predio de la Villa Olímpica, el territorio de la Villa 20 es un espacio donde el planeamiento urbano viene batallando desde hace más de cincuenta años. En este sector se testearon diferentes políticas públicas concebidas para intervenir en los espacios informales. Los planes de erradicación de villas de los ańos sesenta y setenta (véase Massidda, 2018; Snitcofsky, 2012), las cooperativas de autoconstrucción de los ochenta y los programas sociales de los noventa dan cuenta del amplio repertorio tecnológico con el que los planificadores intervinieron las villas miseria, obteniendo en general escasos resultados concretos (Elinbaum \& Massidda, 2020). Esta lenta exploración instrumental de prueba y error fue dramáticamente suspendida en 2014, tras la toma del "cementerio de autos" de la Policía Federal y los mediáticos desalojos del predio de 12 hectáreas adyacente a la Villa 20. Se trata de una parcela que el Gobierno de la Ciudad intentaba privatizar, tal como se sugería en la primera versión del Plan Maestro para la Comuna 8. Este episodio movilizó la aprobación de la Ley de Urbanización de la Villa 20 (Ley 1770), marcando un 
hito en la normativa urbanística e inaugurando un nuevo estilo de planeamiento en la ciudad.

En 2015, a partir de este antecedente y gracias a la convergencia de la coalición política "Cambiemos" en el gobierno nacional, en el provincial y en el de la CABA, se emprendió de forma decidida y acelerada (con enormes recursos técnicos y presupuestarios) la regularización del territorio de las villas miseria de la ciudad. Así, a lo largo de 2016 se consolidó en la Villa 20 el espacio de negociación denominado Mesa de Gestión Participativa. Esta mesa dio lugar al Proyecto Integral de Reurbanización (PIRU) que tiene como objetivo la integración urbana, habitacional y socioeconómica del barrio, y cuyo contenido temático se establece en la Ley 5705 (Figura 3 y Figura 4). En términos instrumentales, el PIRU tiene un alcance amplio, casi omnicomprensivo, que permite construir y mejorar viviendas, abrir calles y proveer servicios y equipamientos (Motta \& Almansi, 2017). Si bien este proyecto de reurbanización no tiene competencias para regularizar el suelo, puede considerarse como un primer paso para "normalizar" el espacio residencial y convertirlo en espacio inmobiliario. En este sentido, el aspecto más innovador del plan radica en la implementación de un relevamiento ad hoc (Relevamiento Socio Espacial, RELSE), concebido para registrar las obras de reforma o reconstrucción de las viviendas afectadas por la apertura de calles, y para tramitar una ficha en la que se relevan los alojamientos de forma muy similar a una mensura catastral.

FIgURA 3 | Plano de regulación de la Villa 20 a través de la delimitación de tres zonas

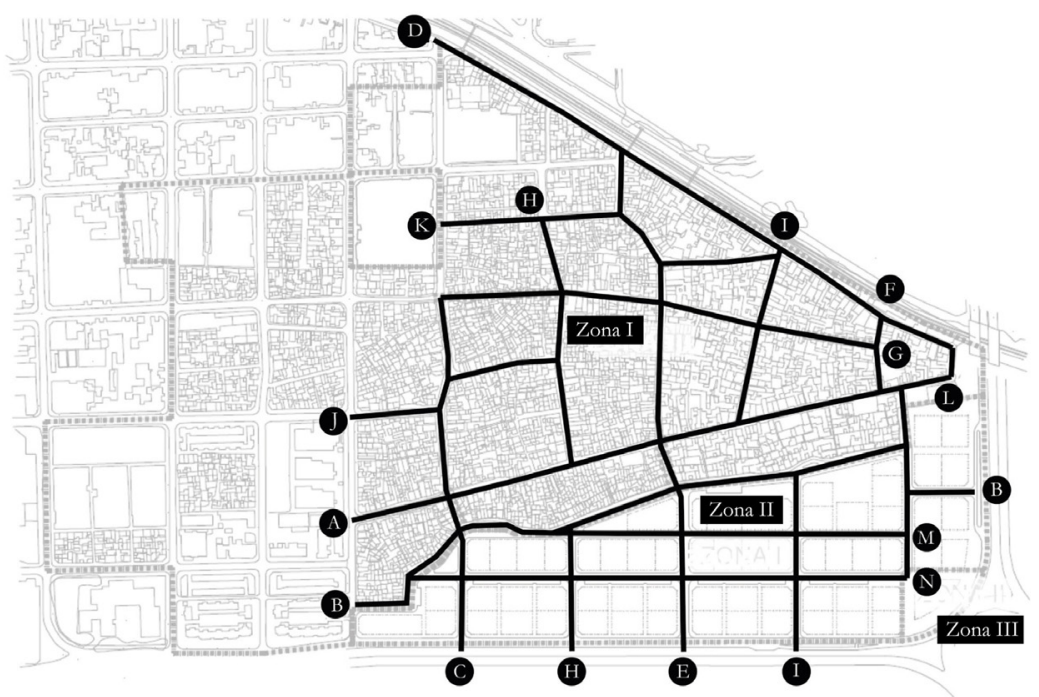

FUENTE: LEY 5705 DE LA CABA 
FIgura 4 | Plano de proyecto de la Villa 20. Red de calles y delimitación de manzanas tipo

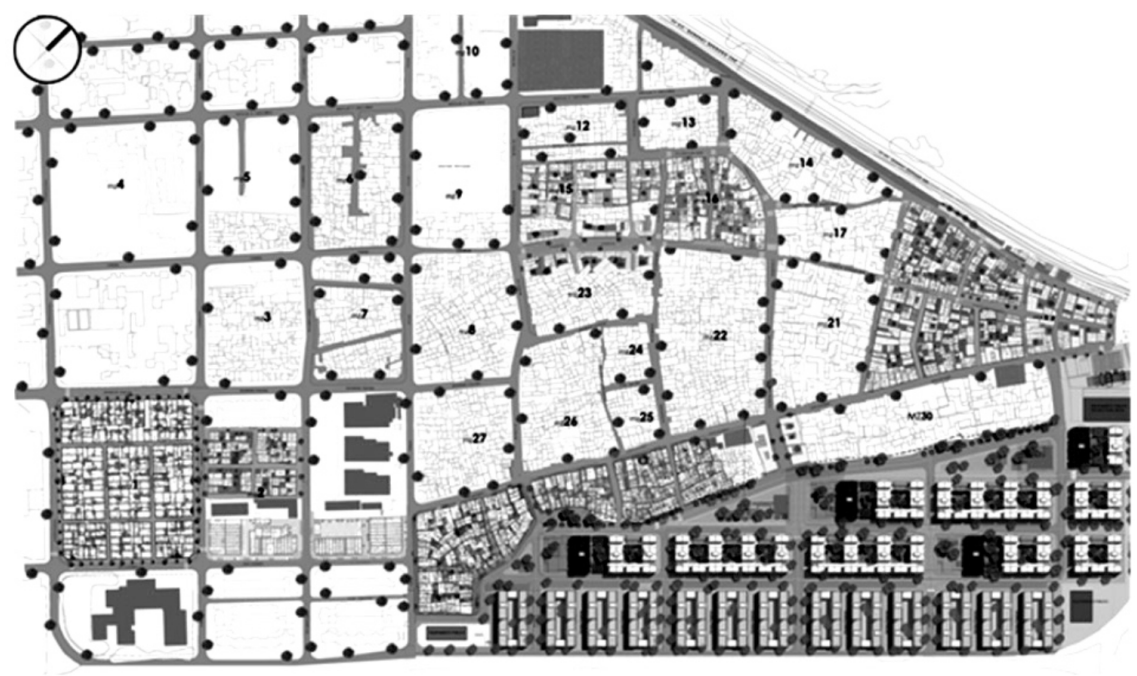

FUENTE: MOTTA Y ALMANSI (20I7)

La cortina y la isla

Además de los cambios en los marcos instrumentales del planeamiento urbano, en el análisis del proyecto de las dos villas se considera también la singularidad de las dinámicas territoriales. En particular, se observan los ciclos de inversión y regulación que, como señalaba Massey (1985), están sujetos a los espacios selectivos y con geometrías variables de la intervención estatal. En este sentido, los primeros límites del sudoeste porteño ya pueden deducirse a partir de la demarcación del área administrativa municipal de Buenos Aires realizada a finales del siglo xIx. Frente a la concepción isótropa y monofuncional del territorio ofrecido casi por completo a la renta inmobiliaria, este sector, difuso en sus fronteras, quedó determinado de hecho por las condiciones topográficas desfavorables para el desarrollo residencial. Así, paulatinamente, el sudoeste porteńo devino en el basurero de la ciudad, un lugar anegado que el Estado destinó a las actividades transitorias e indeseables, como los vertederos, y a la concentración sistemática de los pobres urbanos. Los diferentes ejercicios de planeamiento iniciados en la década del veinte del siglo pasado reafirman estos límites físicos mediante el esquematismo modernista de las actividades segregadas introducidas por Le Corbusier en su propuesta del Plan Director de 1937. Posteriormente, estos límites se expresan de forma más cabal a través del Plan de Autopistas Urbanas implementado por el gobierno militar a mediados de los setenta.

Las huellas de los diferentes bordes y fronteras que conformaron el sudoeste porteño no solo definieron el trapecio que hoy delimita la Comuna 8 , sino que además dan cuenta de la organización histórica y jerarquizada del territorio. En este sentido, la conformación urbana de Buenos Aires se caracterizó, sobre todo, por la 
tajante división espacial del trabajo. En la primera delimitación municipal de 1904, salvo por el puerto y la aduana, se excluyeron las actividades de los sectores primario y secundario, perfilando el desarrollo urbano de Buenos Aires como un espacio de consumo y dependencia, donde "lo productivo" está en otro lado, ya sea en la pampa agropecuaria o en la Europa industrial. Dentro de este proceso, cabe señalar algunos paréntesis en los cuales se intentó industrializar el país y su ciudad capital. El primer gobierno de Perón, a mediados del siglo pasado, imaginó Buenos Aires como una ciudad fordista, y su lado sudoeste como uno de los grandes pulmones verdes que demandaban, como contraparte, las áreas donde se concentrarían las fábricas. Si bien los objetivos de las políticas desarrollistas nunca se completaron, lograron reforzar en el imaginario porteño el norte burgués y el sur obrero. Como señala Gorelik (1998), esta representación urbana ya se venía gestando desde antes; por ejemplo, en el anhelo de equilibrio compensatorio entre el Parque 3 de Febrero, en el norte, y los parques del sur, junto al Riachuelo, que ya figuraban en el Proyecto Orgánico de 1925 (Plan Noel-Forestier) y que apelaban al conocido modelo de París, con el espejo entre el Bois de Boulogne (del lado burgués) y el Bois de Vincennes (del lado obrero). A partir de la década del setenta, frustrado el segundo intento de sustitución de importaciones impulsado por el gobierno de Arturo Frondizi, se retomó el modelo agroexportador, reactivándose el proceso de urbanización rentista, monofuncional y dependiente. A partir de los noventa, esta lógica se exacerbó con la aprobación del estatuto de autonomía de Buenos Aires que rescató a la "Ciudad" del magma de pobreza del conurbano, ubicándola en el mapa de las capitales globales y en la lógica de las reestructuraciones urbanas que demanda la emergente economía del conocimiento y los servicios. En este nuevo escenario, a partir de 2008, el Gobierno de la Ciudad inició un proceso de delimitación de distritos económicos para promover las industrias creativas. Se trata de una nueva capa de regulación, a través de la cual se tematizó a la Comuna 8 como "distrito de los deportes", preparando el terreno legal para pergeńar nuevas opciones de desarrollo inmobiliario, como la Villa Olímpica -y la posibilidad de reurbanizar la Villa 20-, en el marco de los Juegos Olímpicos de la Juventud de 2018 (Figura 5).

Además del dinamismo de los bordes y las escalas territoriales, el carácter socioespacial de la Comuna 8 se refiere a su condición de lugar, expresada en sus particularidades morfológicas y en los modos en que tal condición se interpreta en el proyecto de cada villa. Esto último se evidencia en las estrategias específicas relacionadas al espacio público, al trazado viario, a la alineación de las manzanas, al parcelamiento y a la tipología residencial.

Por su parte, el Proyecto Integral de Reurbanización de la Villa 20 se inició en paralelo a la ejecución del barrio de vivienda social Papa Francisco, construido sobre los terrenos donde se llevó a cabo la toma de 2014. Sus bloques alineados sobre la avenida Fernández de la Cruz conforman una fachada regular que deja a sus espaldas el paisaje precario de la Villa 20. Detrás de esa cortina, el Gobierno de la Ciudad montó un verdadero laboratorio urbanístico para intervenir y transformar este espacio informal, atándolo con el tejido circundante. De este modo, el proyecto de reurbanización se enmarca en una renovación urbana más amplia que vincula las dos villas, la Veinte y la Olímpica, preparando el terreno para incorporar las 
heterotopías en la lógica del espacio estatal. Esto no significa que los espacios informales de la Comuna 8 no hayan estado previamente mercantilizados, aunque su desarrollo por fuera del marco institucional y normativo del código urbano impidió que el Estado pueda regularlos, gravarlos o expropiarlos.

FIGURA 5 | Bordes y barreras del nuevo espacio estatal que reestructura el territorio de la Comuna 8



FUENTE: ELABORACIÓN PROPIA, CON BASE EN LA DOCUMENTACIÓN DEL GOBIERNO DE LA CIUDAD Y EL RELEVAMIENTO DE CAMPO

Antes de ejecutar las obras de reurbanización, el proyecto se inició delimitando de manera escrupulosa el espacio informal de la Villa 20, aislándolo como si estuviese en cuarentena y distinguiendo tres sectores caracterizados por su morfología (consolidada, maciza y vacía), como un primer paso para establecer tres áreas de intervención institucionalizadas en el marco de la Ley 5705 (Figura 6: 6.1 y 6.2). En segundo lugar, se realizaron aperturas en el macizo edificado para darle continuidad a la red de caminos que fijan el perímetro de las manzanas y modulan el espacio continuo de la villa, para atarlo a las calles del entorno (Figura 6: 6.3, 6.4 y 6.5). Sin embargo, más allá de las obras, la acción más estratégica del proyecto de reurbanización ha sido el Relevamiento Socio Espacial (RELSE), implementado de forma ad hoc para complementar la información censal previamente registrada por el Instituto Nacional de Estadística y Censos (INDEC). Realizado sobre algunas manzanas piloto, el RELSE se utilizó como referencia para congelar la estructura 
parcelaria y distinguir las unidades de vivienda dentro del aglomerado de edificios irregulares y superpuestos, donde los pasajes de acceso y los alojamientos se encuentran totalmente imbricados (Figura 6: 6.6 y 6.7). Este proceso de relevamiento puede considerarse como un avance de la regularización dominial, si se considera que, para cada vivienda, se deja constancia no solo de la cantidad de personas y hogares, sino también de los elementos edilicios (estructura, cerramientos, solados, cubiertas e instalaciones), los niveles de habitabilidad, las dimensiones y la distribución espacial interna (Figura 6.8). De este modo, cada ficha del relevamiento funciona casi como una protomensura, certificada por el técnico que releva y avalada con la firma del ocupante-titular.

FIGURA 6 | Análisis de las intervenciones del proyecto de la Villa 20
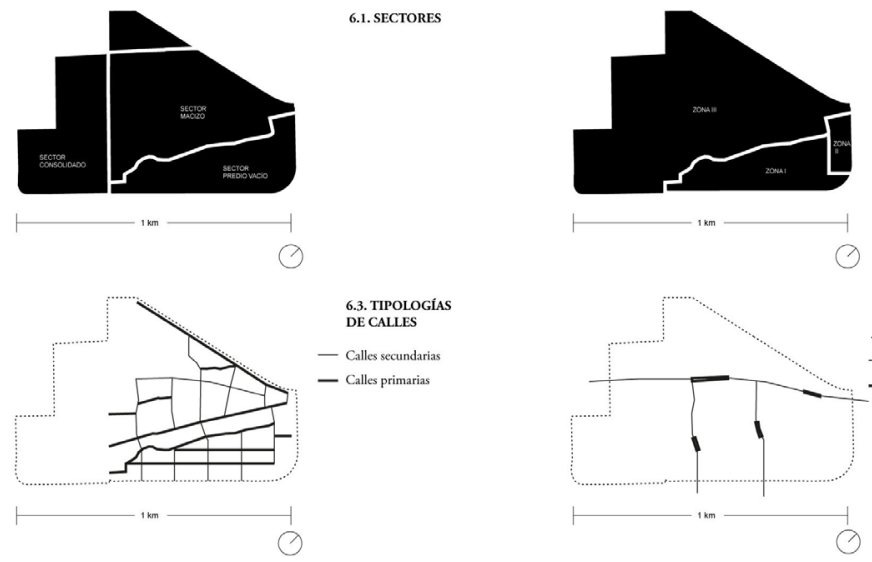

6.2. ZONIFICACIÓN LEY


FUENTE: ELABORACIÓN PROPIA, CON BASE EN LA DOCUMENTACIÓN DEL GOBIERNO DE LA CIUDAD 
Del otro lado de la cortina que oculta la Villa 20, la otra villa, la Olímpica, se proyectó esencialmente como una isla. Ubicado como una bisagra entre el Golf Club José Jurado y el Parque de la Ciudad, el nuevo barrio se concibió como un núcleo independiente, al margen del paisaje degradado de la Comuna 8. Por eso, la delimitación y modulación de sus espacios abiertos y construidos no parte de los elementos del entorno, sino del replanteo de un patrón regular de calles y manzanas cuadradas con esquinas achaflanadas muy similares a las del ensanche de Cerdà en Barcelona (Figura 7: 7.1 y 7.2). Al igual que las ciudades creadas a partir de las Leyes de Indias, el nuevo manzanero parecería colonizar por etapas un territorio de supermanzanas en apariencia vacío e inhóspito (Figura 7: 7.3 y 7.4). Ni la memoria del proyecto ni las bases del concurso de arquitectura ofrecen argumentos sobre esta opción morfológica. Podría decirse que la propuesta evita la mímesis con el paisaje de bloques aislados, asociado a la vivienda popular, ofreciendo, con el nuevo tejido de manzanas, un giro morfológico -y social- en la concepción del espacio estatal del sudoeste porteño. En cambio, los indicadores urbanísticos aparecen claramente especificados mediante una serie de regulaciones edilicias, denominadas en las bases del concurso como "tejido definido por variantes morfológicas" (Figura 8). Esta guía de composición fija requerimientos formales y generales tendientes a que los diferentes emprendimientos consoliden el anillo perimetral alineado a la calle, la altura regular de las cornisas y el centro de manzana (Figura 7.5). Asimismo, establece una serie de condiciones más específicas y sutiles que conciernen al diseño de las fachadas y que tienen como fin recrear "las situaciones de heterogeneidad y complejidad" que caracterizan el paisaje urbano de Buenos Aires (Sociedad Central de Arquitectos, SCA, 2014). Visto de otro modo, se trata de que este pedacito de Barcelona en Buenos Aires simule el paso del tiempo a través de la tematización de los tejidos decimonónicos, soslayando la impronta de masividad y urgencia en la ejecución -la mano del Estado- que denotan los grandes conjuntos de vivienda social recién inaugurados.

El cuidado en la concepción del espacio estatal de la Villa Olímpica involucra no solo la estructura parcelaria y la morfología urbana (Figura 7.7), sino también al tipo de vivienda. En este sentido, el proyecto incorpora algunos rasgos del "inmueble de renta" decimonónico europeo, tales como las medianeras, las viviendas enfrentadas a la línea municipal o al centro de manzana y los bloques intermedios separados por patios interiores, en general de dimensiones "técnicas" de ventilación e iluminación. Esta tipología, emparentada con la del petit hotel porteño (cf. Diez, 1996), reproduce objetivos especulativos que condicionan, entre otras cosas, a que la mitad de las viviendas se enfrenten a los patios interiores (Figura 7.6). Sin ánimo de entrar en la crítica arquitectónica, se trata de una disposición inentendible, si consideramos que todo el proyecto se ejecuta sobre un predio sin grandes accidentes infraestructurales o topográficos. Además, si bien las reglas "morfológicas" obligan a preservar el centro de manzana vacío, como un intento voluntarista de construcción de un espacio comunitario, el tipo dominante de la mayoría de las viviendas - unidades de dos y tres ambientes (Figura 7.8) - excluyen automáticamente a las "familias numerosas" características del barrio, que claramente no calzan en "ese" proyecto comunitario. 
La producción del espacio estatal en los proyectos analizados se evidencia también a partir de la noción de red. Este concepto es importante para entender hasta qué punto los proyectos fueron pensados como dinamizadores e irradiadores de centralidad, en función de la estrategia de atraer inversiones privadas e incorporar este territorio al tejido circundante y también al mercado inmobiliario formal. Así, los planes parciales de las dos villas operan en dos sentidos. En el caso del proyecto de reurbanización de la Villa 20, la nueva infraestructura de calles moduladas y atadas a la trama regular del tejido circundante contiene y fragmenta la red de pasajes internos, desactivando paulatinamente su carácter rizomático. Mientras, en el caso de la Villa Olímpica se opta por la estrategia del cambio de escala (Figura 8). El proyecto intenta pasar de la grilla de supermanzanas modernistas - del espacio público concebido como espacio técnico para la movilidad-, a la escala más menuda de las manzanas urbanas (Figura 7: 7.2 y 7.3), pero manteniendo siempre la distancia física y social -e incluso paisajística y simbólica- con respecto a las villas miseria y a los polígonos de vivienda social circundantes, como los de Soldati y Lugano (Figura 5).

FIGURA 7 | Análisis socioespacial del proyecto de la Villa Olímpica
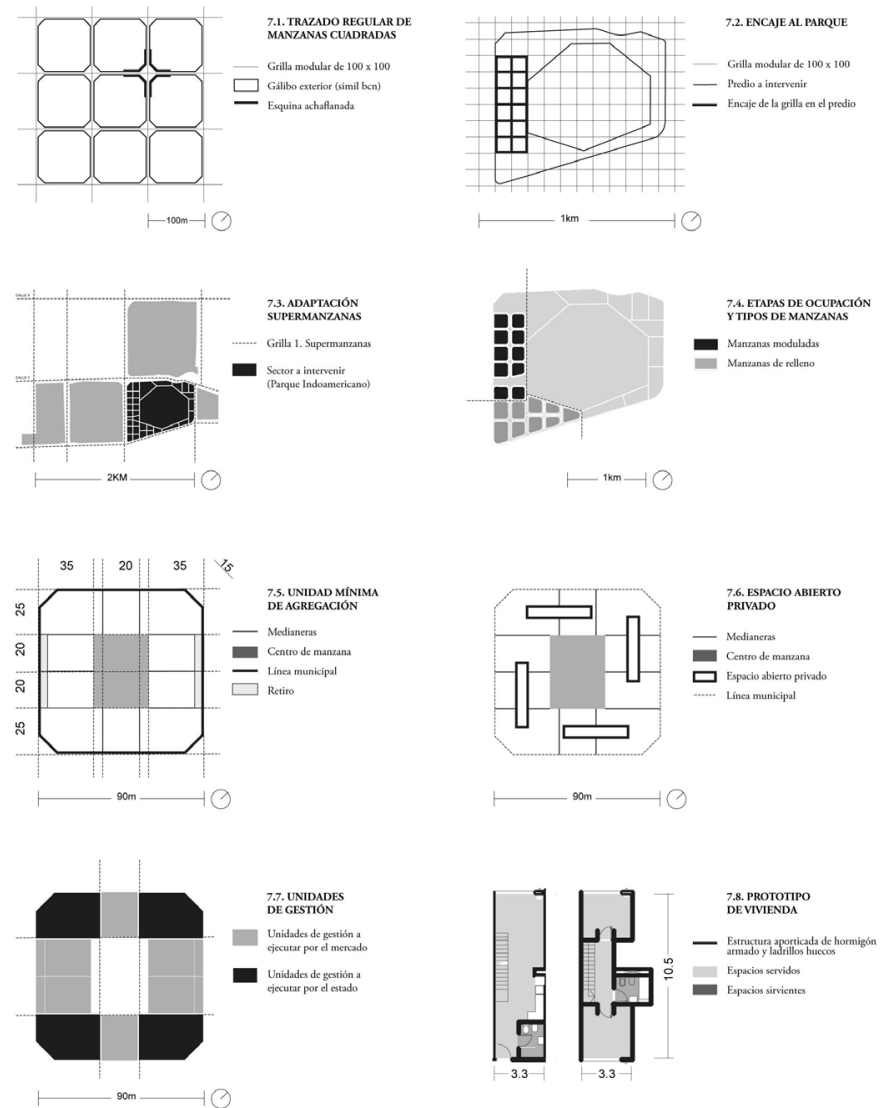

FUENTE: ELABORACIÓN PROPIA, CON BASE EN LA DOCUMENTACIÓN DEL GOBIERNO DE LA CIUDAD Y EL RELEVAMIENTO DE CAMPO 
FIGURA 8 | Tejido definido por variantes morfológicas. Bases del concurso de la Villa Olímpica

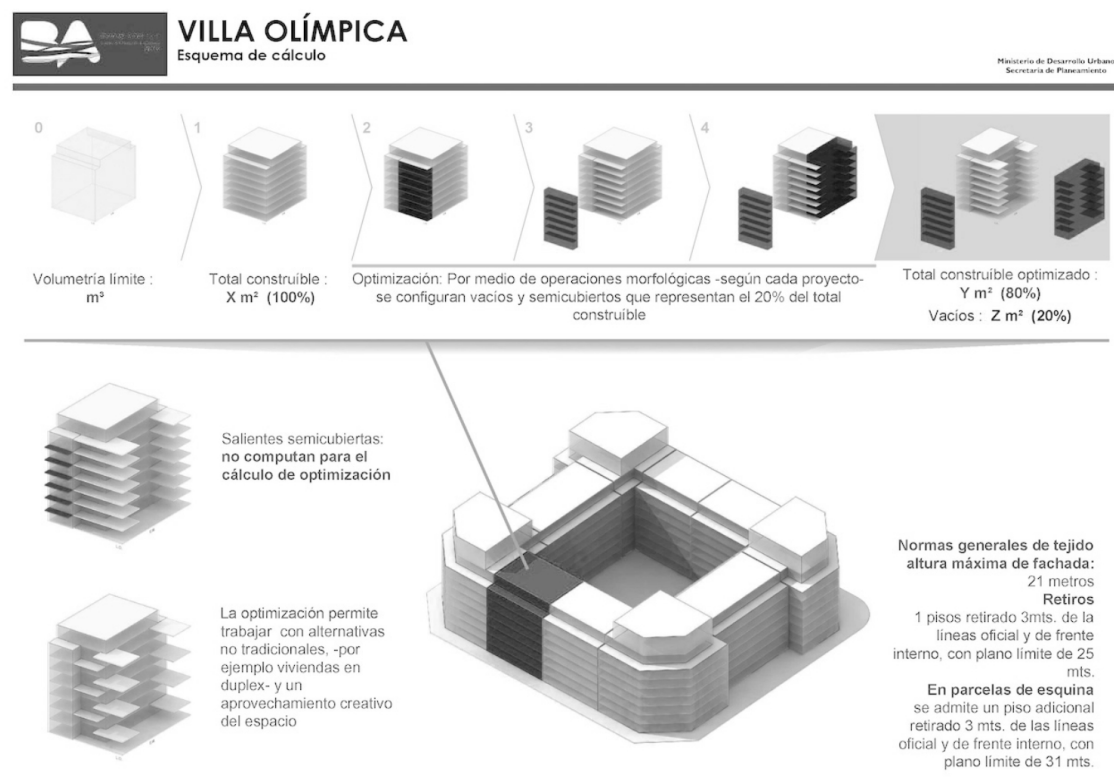

FUENTE: SOCIEDAD CENTRAL DE ARQUitectos (SCA), 20 I 6

\section{Conclusiones}

Las heterotopías urbanas, los lugares que interrumpen la aparente continuidad y normalidad de la ciudad, han sido estudiadas desde sus aspectos históricos, culturales y sociológicos. Menos atención han recibido por su implicancia en la producción del espacio estatal y, en particular, en los marcos codificados y normativos del planeamiento urbano. A través del estudio comparado de dos planes parciales porteńos concurrentes en la Comuna 8, uno implementado para desarrollar la Villa Olímpica y el otro para reurbanizar la Villa 20, en este artículo se indagó en los modos en que los urbanistas conciben e intervienen las heterotopías para incorporarlas en la territorialidad de los Estados capitalistas, a través del entramado de proyectos y estrategias. Tal como se definen en este artículo, los proyectos proveen la unidad, la estabilidad y la coherencia de la estructura del espacio estatal, produciendo "efectos de estado" que transmiten la imagen formalista de sus jurisdicciones y niveles de planeamiento y omiten, al mismo tiempo, los históricos desequilibrios socioespaciales. En cambio, las estrategias estatales apuntan a movilizar el circuito del capital a través de formas selectivas e innovadoras de intervención socioeconómica. Dicho de otro modo, los proyectos ofrecen una imagen horizontal del territorio, mientras que las estrategias lo inclinan, y así sucesivamente.

El estudio de los dos casos mencionados permitió, además, reflexionar acerca de la especificidad de los grandes proyectos urbanos y de las villas miseria, dos de las 
heterotopías paradigmáticas de las metrópolis latinoamericanas, y clarificar algunas relaciones no tan evidentes, complementarias y dinámicas, entre diferentes territorios, lugares, escalas, vectores de desarrollo y ciclos de inversión y de regulación urbanística. Relaciones que suelen ser soslayadas no solo por las investigaciones acotadas a los "enclaves", sino también por los objetivos trascendentales de las políticas urbanas pretendidamente "integrales". El análisis de estos vínculos evidenció una serie de novedades en la producción de la territorialidad del Estado, las cuales se evidencian, en particular, a partir de dos dimensiones básicas del planeamiento urbano: la instrumental y la socioespacial (Tabla 1).

En cuanto a la dimensión instrumental, los casos mostraron que, cuando se trata de abordar las heterotopías urbanas, los planes parciales o de sector se convierten en verdaderos laboratorios urbanísticos. Durante el proceso de planeamiento se exploraron dos modalidades de intervención selectiva. La primera se refiere al relevamiento minucioso de las construcciones informales y de los déficits urbanos, como guía para ejecutar las infraestructuras básicas y como una oportunidad para instaurar un marco de negociación tendiente a regularizar, normalizar y fijar la dinámica expansiva de las villas miseria. La segunda modalidad de intervención está más emparentada con algunos de los mecanismos ya probadas en los grandes proyectos urbanos, como la creación de indicadores excepcionales, la venta de suelo público, la creación de unidades de gestión a medida de las grandes empresas constructoras y la articulación del desarrollo inmobiliario con la realización de megaeventos. Más novedosa es la iniciativa por parte del gobierno local, la de asumir el riesgo de la promoción inmobiliaria para ejecutar y comercializar él mismo prácticamente la mitad del parque de viviendas, activando y acelerando el proceso de renovación urbana. Cabe señalar que los dos frentes de intervención de esta maniobra unitaria están ligados más sutilmente a través de la articulación estratégica de sus respectivas partidas presupuestarias, en la medida en que la reurbanización de la Villa 20 está sujeta, en parte, al dinero obtenido por las ventas de las unidades de gestión de la Villa Olímpica (Breckner et al., 2017).

Estas reestructuraciones instrumentales permiten reflexionar acerca de la segunda dimensión de análisis, la socioespacial, a través de la cual se pudo evidenciar cómo los casos estudiados complementan sus enfoques proyectuales y estratégicos. El primero apunta a delimitar, aislar y ocultar los espacios informales para modularlos desde adentro, atándolos a la trama regular de la ciudad. Se trata, de este modo, de reducir el carácter rizomático de las villas miseria, sus circulaciones y alojamientos solapados. Al mismo tiempo, se trata de congelar el espacio doméstico para convertirlo paulatinamente en espacio inmobiliario. De forma inversa, pero complementaria, el segundo enfoque proyectual y estratégico pretende superponer un patrón preconcebido, convencional y familiar, en un territorio supuestamente vacío y hostil. Así, a través de la recreación de espacios residenciales "normales", como los de las manzanas cerradas y los inmuebles de renta decimonónicos, se procura diferenciar los nuevos emprendimientos inmobiliarios con respecto al tradicional paisaje que conforman las villas miseria y los bloques aislados de los conjuntos de vivienda social. 
TABLA I | Síntesis del análisis cruzado de los casos en términos instrumentales y socioespaciales, en los cuales se distingue el sentido proyectual del estratégico

\begin{tabular}{|c|c|c|c|c|}
\hline \multirow{2}{*}{ DIMENSIÓN } & \multicolumn{2}{|c|}{ VILLA 20} & \multicolumn{2}{|c|}{ VILLA OLÍMPICA } \\
\hline & PROYECTO & ESTRATEGIA & PROYECTO & ESTRATEGIA \\
\hline Instrumental & $\begin{array}{l}\text { Parcelar y modular } \\
\text { el espacio de la villa } \\
\text { miseria, fijando el } \\
\text { perímetro de las } \\
\text { manzanas, el espacio } \\
\text { público -y el priva- } \\
\text { do-, precisando las } \\
\text { medianeras y ejecu- } \\
\text { tando las infraestruc- } \\
\text { turas básicas. }\end{array}$ & $\begin{array}{l}\text { Instaurar un marco } \\
\text { de negociación y } \\
\text { regularización de la } \\
\text { dinámica autóno- } \\
\text { ma de los espacios } \\
\text { informales, para } \\
\text { convertirlos paulati- } \\
\text { namente en espacios } \\
\text { inmobiliarios. }\end{array}$ & $\begin{array}{l}\text { Movilizar la renova- } \\
\text { ción urbana, atando } \\
\text { la venta del suelo } \\
\text { público a los megae- } \\
\text { ventos y calibrando } \\
\text { el tamaño de las uni- } \\
\text { dades de gestión de } \\
\text { la vivienda pública } \\
\text { a la capacidad de las } \\
\text { empresas construc- } \\
\text { toras. }\end{array}$ & $\begin{array}{l}\text { Convertir al Estado } \\
\text { en desarrollador, } \\
\text { asumiendo el riesgo } \\
\text { de la inversión en } \\
\text { infraestructuras y la } \\
\text { promoción inmobi- } \\
\text { liaria, para activar y } \\
\text { acelerar el proceso de } \\
\text { renovación urbana. }\end{array}$ \\
\hline Socioespacial & $\begin{array}{l}\text { Contener el carácter } \\
\text { expansivo y rizomá- } \\
\text { tico de los espacios } \\
\text { informales dentro } \\
\text { del perímetro de las } \\
\text { nuevas manzanas, } \\
\text { atándolos a la conti- } \\
\text { nuidad y regularidad } \\
\text { de los tejidos circun- } \\
\text { dantes. }\end{array}$ & $\begin{array}{l}\text { Gestionar los tiem- } \\
\text { pos de la renovación } \\
\text { urbana, vinculando } \\
\text { el presupuesto de la } \\
\text { reurbanización de } \\
\text { las villas miseria a la } \\
\text { venta de las unidades } \\
\text { de los grandes pro- } \\
\text { yectos urbanos. }\end{array}$ & $\begin{array}{l}\text { Instaurar una morfo- } \\
\text { logía y una tipología } \\
\text { patricia -tradicional } \\
\text { y clasista- para co- } \\
\text { lonizar y vender un } \\
\text { territorio concebido } \\
\text { como inhóspito y } \\
\text { vacío. }\end{array}$ & $\begin{array}{l}\text { Diferenciar la } \\
\text { residencia nueva del } \\
\text { carácter socioespa- } \\
\text { cial peyorativo de } \\
\text { los conjuntos de } \\
\text { vivienda social, para } \\
\text { inducir el proceso de } \\
\text { gentrificación. }\end{array}$ \\
\hline
\end{tabular}

FUENTE: ELABORACIÓN PROPIA

La diferenciación socioespacial de la Villa Olímpica, un barrio construido ex novo que podríamos considerar como un miniensanche, abre la oportunidad de reflexionar, de forma más general, sobre la vigencia de las normas urbanísticas convencionales, considerando además la reciente revisión del código urbano de Buenos Aires como un episodio significativo en relación con los temas tratados en este artículo. La elección de una morfología tradicional (la manzana cuadrada en forma de corona), además de reavivar el anhelo patricio de convertir Buenos Aires en París y, ahora, en Barcelona, no hace más que responder al objetivo de la especulación rentista que guio su desarrollo urbano durante más de un siglo (Elinbaum, 2019). La lógica del máximo aprovechamiento edilicio no solo condiciona la habitabilidad de las viviendas, más o menos favorecidas por el corsé de la línea municipal y las medianeras, sino que, además, reproduce otro objetivo: la diferenciación clasista que otorga el estatus y la meritocracia socioespacial que se proyecta de la forma urbana al tipo de vivienda y viceversa. Estas regulaciones urbanísticas mezquinas (que, entre otras cosas, determinan que más de la mitad de las viviendas que se construyen en la CABA se orienten hacia los patios de "aire y luz", concebidos con la lógica de los espacios técnicos, como la del hueco del ascensor o la del ducto para la basura) se suelen justificar a través de los discursos que, de forma supersticiosa, promueven los valores de la mixtura morfológica, la compacidad y la alta densidad como garantías de la cohesión social. 
Un último tema que involucra a la producción del espacio estatal, abierto a futuras investigaciones, es el de la relación entre la centralidad y la heterotopía. ¿Hasta qué punto las reestructuraciones espaciales, impulsadas por los proyectos de las dos villas, contemplan o inciden en la centralidad urbana? ¿En qué medida el planeamiento urbano influye en la producción de los espacios de centralidad? La incidencia de la inversión o la regulación estatal en la intensidad de las actividades urbanas, frente a otros factores más espontáneos, es algo aún difícil de probar. Por lo pronto, a la luz de la respuesta de los pocos locatarios de las viviendas de la Villa Olímpica y los desarrolladores privados que deberían completar la ejecución del barrio, parecería que las inversiones realizadas por el Estado no son suficientes como para movilizar este proceso de renovación urbana, tanto espacial como social. El tiempo dirá si el cambio de escala de las supermanzanas de la Comuna 8 a las manzanitas de la Villa Olímpica logran ir más allá de la centralidad de enclave para promover la centralidad de cercanía.

\section{Referencias bibliográficas}

Amin, A. (2002). Spatialities of globalisation. Environment and Planning A: Economy and Space, 34(3), 385-399. https://doi.org/10.1068/a3439

Breckner, I., Gerscovich, A., Lehner, J. \& Pape, T. (2017). ¿Urban voids? Buenos Aires-Hamburg. Report of the international workshop in Buenos Aires. HafenCity Universität y Centro de Estudios del Hábitat y la Vivienda (CeHyv), Universidad de Buenos Aires.

Brenner, N. (1998). Between fixity and motion: accumulation, territorial organization and the historical geography of spatial scales. Environment and Planning D: Society and Space, 16(4), 459-481. https://doi.org/10.1068/d160459

Brenner, N. (2000). The urban question: Reflections on Henri Lefebvre, urban theory and the politics of scale. International Journal of Urban and Regional Research, 24(2), 361-378. https://doi.org/10.1111/1468-2427.00234

Brenner, N. (2004). New state spaces: urban governance and the rescaling of statehood. Oxford University Press.

Brenner, N., Jessop, B., Jones, M. \& Macleod, G. (Eds.). (2003). State/Space: A Reader. Blackwell.

Caride, H. \& Dal Castello, D. (2015). Heterotopías. Anales del IAA, 44(2), 9-14. http://www. iaa.fadu.uba.ar/ojs/index.php/anales/article/view/143

Castells, M. (1996). The rise of the network society. Blackwell.

Collinge, C. (1999). Self-organisation of society by scale: a spatial reworking of regulation theory. Environment and Planning D: Society and Space, 17(5), 557-574. https://doi. org/10.1068/d170557

Cresswell, T. (2004). Place: A short introduction. Blackwell. 
Cuenya, B. (2012). Grandes proyectos urbanos como herramientas de creación y captura de plusvalías inmobiliarias. En A. Ziccardi (Comp.), Ciudades del 2010: entre la sociedad del conocimiento y la desigualdad social (pp. 1019-1038). México: Programa Universitario de Estudios sobre la Ciudad (puec), Universidad Nacional Autónoma de México (unam).

Dehaene, M. \& De Cauter, L. (Eds.). (2008). Heterotopia and the city. Public space in a postcivil society. Routledge. https://doi.org/10.4324/9780203089415

Dicken, P., Kelly, P. F., Olds, K. \& Yeung, H. (2001). Chains and networks, territories and scales, towards a relational framework for analysing the global economy. Global Networks, 1(2), 89-112. https://doi.org/10.1111/1471-0374.00007

Diez, F. (1996). Buenos Aires y algunas constantes en las transformaciones urbanas. Universidad de Belgrano, Buenos Aires.

Elinbaum, P. (2019). La ciudad como proyecto estatal. Revista PLOT, dic-ene, 15-19. https:// www.researchgate.net/publication/332031911_La_ciudad_como_proyecto_estatal

Elinbaum, P. \& Massidda, A. (2020). La representación urbanística de los espacios informales. Las villas miseria de Buenos Aires (1947-1965). Bitácora Urbano Territorial, 30(1), 115-126. http://dx.doi.org/10.15446/bitacora.v30n1.70124

Esteban, J. (1998). Elementos de ordenación urbana. Ediciones UPC (Universitat Politècnica de Catalunya).

Faludi, A. (1996). Rationiality, critical rationalism, and planning doctrine. En S. J. Mandelbaum, L. Mazza \& R. W. Burchell (Eds.), Explorations in planning theory (pp. 65-82). Center for Urban Policy Research, Rutgers University.

Faludi, A. \& van der Valk, A. (1994). Rule and order: Dutch planning doctrine in the twentieth century. Kluwer Academic Publishers.

Foucault, M. (1984[1967]). Des espaces autres. Architecture, Mouvement, Continuité, 5, 46-49. https://historiacultural.mpbnet.com.br/pos-modernismo/Des_espaces_autres.pdf

Gil, A., Cosacov, N., Di Virgilio, M., Gil y de Anso, M. L., Guevara, T., Imori, M., Menazzi, M. L., Ostuni, F., Perea, C. M., Perelman, M., Ramos, J. M., Rodríguez, M. F., Paschkes Ronis, M. \& Vitale, P. (2011). Barrios al sur: Villa Lugano, Villa Riachuelo, Mataderos, Parque Patricios y Villa Soldati a través del tiempo. Documentos de Trabajo (Universidad de Buenos Aires. Facultad de Ciencias Sociales), (56). https://dialnet. unirioja.es/servlet/articulo?codigo $=3717979$

Gorelik, A. (1998). La grilla y el parque: espacio público y cultura urbana en Buenos Aires, 18871936. Universidad Nacional de Quilmes.

Grabher, G. (2006). Trading routes, bypasses and risky intersections, mapping the travels of 'networks' between economic sociology and economic geography. Progress in Human Geography, 30(2), 163-189. https://doi.org/10.1191/0309132506ph600oa

Graham, S. \& Marvin, S. (2001). Splintering urbanism: networked infrastructures, technological mobilities and the urban condition. Routledge.

Fernández, O. (2017). Heterotopías urbanas: una mirada foucaultiana a las favelas cariocas. Daimon Revista Internacional de Filosofia, (71), 81-93. https://doi.org/10.6018/ daimon/236681

Hudson, R. (2002). Producing places. Guilford Press. 
Jajamovich, G. (2019). Grandes proyectos urbanos alternativos o alternativas a los grandes proyectos urbanos: una revisión a partir del concepto de just city. Cuadernos de Geografia: Revista Colombiana de Geografia, 28(2), 394-407. https://doi.org/10.15446/ rcdg.v28n2.72090

Jajamovich, G. \& Kozak, D. (2019). Urban megaprojects. En A. Orum (Ed.), The Willey \& Blackwell Encyclopedia of Urban and Regional Studies (pp. 2425-2427). Willey \& Blackwell.

Janoschka, M. (2016). Gentrificación, desplazamiento, desposesión: Procesos urbanos clave en América Latina. Revista INVI, 31(88), 27-71. http://dx.doi.org/10.4067/S071883582016000300002

Jessop, B. (2001). Institutional re(turns) and the strategic-relational approach. Environment and Planning A: Economy and Space, 33(7), 1213-1235. https://doi.org/10.1068/a32183

Jessop, B., Brenner, N. \& Jones, M. (2008). Theorizing sociospatial relations. Environment and Planning D: Society and Space, 26(3), 389-401. https://doi.org/10.1068/d9107

Jessop, B. \& Sum, N. (2006). Beyond the regulation approach: Putting capitalist economies in their place. Edward Elgar.

Lungo, M. (2004). Grandes proyectos urbanos. Una visión general. En M. Lungo (Comp.), Grandes proyectos urbanos. UCA Editores (Universidad Centroamericana "José Simeón Cañas") / Lincoln Institute of Land Policy.

Massey, D. (1984). Spatial divisions of labor: Social structures and the geography of production. Methuen.

Massey, D. (1985). New directions in space. En D. Gregory (Ed.), Social relations and spatial structures (pp. 9-19). Macmillan Education UK.

Massey, D. (1994). Space, place, and gender. University of Minnesota Press.

Massidda, A. (2018). Utopian visions for Buenos Aires shantytowns: Collective imaginaries of housing rights, upgrading and eviction (1956-2013). Bulletin of Latin American Research, 37(2), 144-159. https://doi.org/10.1111/blar.12559

Motta, J. M. \& Almansi, F. (2017). Gestión y planificación por proceso - proyecto para el mejoramiento de villas y asentamientos de gran escala. El caso de la reurbanización de Villa 20 en la caBA. Medio Ambiente y Urbanización, 86(1), 145-168. https://www. ingentaconnect.com/contentone/iieal/meda/2017/00000086/00000001/art00007

Neuman, M. (1998). Does planning need the plan? Journal of the American Planning Association, 64(2), 208-220. https://doi.org/10.1080/01944369808975976

Paasi, A. (2004). Place and region: Looking through the prism of scale. Progress in Human Geography, 28(4), 536-546. https://doi.org/10.1191/0309132504ph502pr

Roitman, A. (2016). La Comuna 8 y los planes urbanos para Buenos Aires. Revista HACHE, (3), 38-65.

Santos, O. (2014). Urban common space, heterotopia and the right to the city: Reflections on the ideas of Henri Lefebvre and David Harvey. Urbe, 6(2), 146-157. https://doi. org/10.7213/urbe.06.002.SE02

Sheppard, E. (2002). Thespacesand times of globalization: place, scale, networks, and positionality. Economic Geography, 78(3), 307-330. https://doi.org/10.1111/j.1944-8287.2002. tb00189.x

Silvestre, G. (2017). O não legado e os Jogos que não foram. A primeira candidatura olímpica do Rio de Janeiro e o imaginário de legado urbano para a cidade. Arquitextos, (17). 
Smith, N. (1995). Remaking scale: Competition and cooperation in prenational and postnational Europe. En H. Eskelinen \& F. Snickars (Eds.), Competitive European Peripheries (pp. 59-74). Springer.

Smolka, M. \& Furtado, F. (2001). Recuperación de plusvalias en América Latina: Alternativas para el desarrollo urbano. P. Universidad Católica de Chile y Lincoln Institute of Land Policy.

Snitcofsky, V. (2012). Clase, territorio e historia en las villas de Buenos Aires (1976-1983). Quid 16: Revista del Área de Estudios Urbanos, 16(2), 46-62. https://dialnet.unirioja. es/servlet/articulo?codigo $=5593372$

Swyngedouw, E. (1997). Neither global nor local: 'glocalization' and the politics of scale. En K. Cox, Spaces of Globalization (pp. 137-166). Guilford Press.

Taylor, P. (2004). The state as container, territoriality in the modern world-system. Progress in Human Geography, 18(2), 151-162. https://doi.org/10.1177/030913259401800202

Tramontani, T. (2010). Heterotopias urbanas: Espaços de poder e estratégias sócio-espaciais dos Sem-Teto no Rio de Janeiro. POLIS, 9(27), 293-313. http://dx.doi.org/10.4067/ S0718-65682010000300013

Valverde, R. (2009). Sobre espaço público e heterotopia. Geosul, 24(48), 7-26. https://doi. org/10.5007/2177-5230.2009v24n48p7

Van Duinen, L. (2004). Planning imagery: The emergence and political acceptance of planning concepts in Dutch national planning. Universiteit van Amsterdam, Faculteit der Maatschappij - en Gedragswetenschappen.

Ziccardi, A. (1977). Políticas de vivienda y movimientos urbanos: El caso de Buenos Aires (19631973). Centro de Estudios Urbanos y Regionales (CEUR), Instituto Torcuato di Tella. 